\title{
Efeito da autocorrelação residual na avaliação genética de cabras para a produção de leite e para o formato da curva de lactação
}

\author{
[Effect of residual autocorrelation in the genetic evaluation of goats for milk yield and the \\ shape of the lactation curve] \\ A.L.P. Melo ${ }^{1}$, R.A. Torres ${ }^{2}$, F. Fonseca e Silva ${ }^{3}$, J.I. Ribeiro Júnior ${ }^{3}$, \\ M.T. Rodrigues ${ }^{2}$, G.R.O. Menezes ${ }^{1}$ \\ ${ }^{1}$ Aluna de pós-graduação - UFV - Viçosa, MG
${ }^{2}$ Departamento de Zootecnia - UFV - Viçosa, MG
}

RESUMO

Avaliou-se o efeito da autocorrelação residual sobre a qualidade das estimativas dos parâmetros genéticos para produção total de leite (PL) e para os coeficientes a, b e c do modelo de Wood e, consequentemente, sobre a classificação dos animais para estas características. O modelo de Wood foi ajustado às lactações de cabras considerando-se três situações de estrutura residual: (EI) - erros independentes, (AR1) - erros autorregressivos de primeira ordem e (EI - AR1) - erros AR1 somente para as lactações que apresentaram autocorrelação residual significativa e EI para as demais. As estimativas dos coeficientes a, b e c e PL foram utilizadas como variáveis dependentes em um modelo animal multicaracterístico, o qual incluiu os efeitos aleatórios de animal e de ambiente permanente e os efeitos fixos de grupo contemporâneo, número de crias por parto, grupos genéticos e ordem de parto, além das covariáveis duração da lactação e a idade da cabra ao parto. As diferentes estruturas residuais afetaram as estimativas dos parâmetros genéticos e as classificações dos animais para as características estudadas. Portanto, a seleção dos animais pode ser modificada pela presença da autocorrelação residual, sendo a estrutura EIAR1 uma alternativa para contornar este problema.

Palavras-chave: herdabilidade, modelo animal, repetibilidade, resíduos autorregressivos

\begin{abstract}
The objective of this study was to evaluate the residual autocorrelation effect on the estimates of genetic parameters for total milk yield (MY) and the coefficients $a, b$ and $c$ of Wood's model and, consequently, the animal's classification for these traits. Wood's model was fitted to the lactation of goats considering three cases of residual structure: (IE) - independent errors, (AR1) - first order autoregressive errors, and (IE - AR1) - AR1 errors only for lactations that had significant residual autocorrelation and IE for others. Estimates of the coefficients $a, b$ and $c$ and $P L$ were used as dependent variables in a multivariate animal model, which included the random effects of animal and permanent environmental and fixed effects of contemporary group, number of foals per birth, genetic groups and order of calving, besides the covariates of lactation length (linear) and age at parturition (linear and quadratic). The different residual structures affected the estimates of genetic parameters and the classification of animals for the traits studied. Therefore, the selection of animals can be modified with the presence of residual autocorrelation and the structure IE-AR1 is presented as an alternative to solve this problem.
\end{abstract}

Keywords: animal model, autoregressive errors, heritability, repeatability

Recebido em 23 de maio de 2010

Aceito em 14 de abril de 2011

E-mail: analupuerro@yahoo.com.br 


\section{INTRODUÇÃO}

A melhoria da capacidade produtiva dos animais, sob o ponto de vista genético, consiste em um dos pontos chaves do sistema de produção, já que animais geneticamente superiores apresentam maior capacidade de responder às melhorias no sistema produtivo. No entanto, no Brasil, ainda há poucos programas de melhoramento para caprinos leiteiros. Nesse contexto, estudos de metodologias que visem maximizar a eficiência da avaliação genética de caprinos leiteiros são de grande importância.

A análise da forma da curva de lactação consiste em uma metodologia que pode contribuir bastante para a eficiência da avaliação genética destes animais, pois, além de estar relacionada com a produção de leite em si, permite fazer considerações sobre aspectos como o pico de produção e a persistência da lactação, os quais podem ser utilizados em programas de seleção. Assim, o estudo da forma da curva de lactação de cabras possibilitaria a seleção dos animais com o formato da curva mais eficiente para o sistema produtivo.

Segundo Oliveira et al. (2007), as curvas de lactação são estudadas mediante uso de modelos matemáticos e são importantes para o estabelecimento de estratégias capazes de otimizar a seleção e a busca de genótipos mais eficientes e rentáveis para o produtor. Os modelos são úteis para se estimar a produção de leite na lactação a partir de resultados parciais e para se fazer projeções de acordo com a extensão do período de lactação, propiciando a tomada de decisão quanto à permanência de animais no rebanho.

Em estudos com vacas leiteiras, Wood (1967) e Johansson e Rendel (1972) constataram que é economicamente preferível ter um animal com produção de leite moderada e de forma constante durante toda a lactação do que um que produza grande quantidade de leite até o pico, logo após, e tenha queda acentuada. Vacas mais persistentes requerem menor quantidade de alimento concentrado - do que as com alta produção no pico, porém menos persistentes. Aquelas estão sujeitas ao menor estresse, o que reduz a incidência de problemas reprodutivos e de doenças metabólicas (Sölkner e Fuchs, 1987; Grossman et al., 1999).
Em um estudo sobre o ajuste de funções matemáticas à curva de lactação em caprinos, Guimarães et al. (2006) ressaltaram que a determinação dos valores genéticos dos coeficientes do modelo usado para descrever curvas de lactação é um fator importante, uma vez que tais coeficientes são responsáveis pela determinação da forma dessas curvas e, consequentemente, pela eficiência da produção de leite.

Uma alternativa de análise da curva de lactação consiste na utilização dos coeficientes dos modelos utilizados para descrever tais curvas como características de interesse em programas de seleção. Dentre estes modelos, pode-se citar o de Wood (1967), cujos coeficientes apresentam interpretação biológica como produção inicial (a) e taxas de ascensão (b) e de decréscimo (c) da produção, respectivamente, antes e após o pico.

No entanto, em estudos que envolveram dados longitudinais como os de curvas de lactação, devido ao fato de um mesmo indivíduo ser repetidamente avaliado ao longo do tempo, muitas vezes a pressuposição de independência dos erros assumida no modelo de regressão adotado não é atendida, levando à ocorrência de autocorrelação residual. Esta pode implicar na obtenção de estimativas viesadas para os coeficientes do modelo e subestimação de suas variâncias (Souza, 1998). Em um estudo sobre curvas de lactação individuais de vacas da raça Caracu, Faro e Albuquerque (2002) constataram, ao ajustarem a função gama incompleta, que 56,5\% das lactações apresentaram autocorrelação residual positiva. A modelagem da autocorrelação residual pode melhorar significativamente $\mathrm{o}$ ajuste do modelo de regressão, e este procedimento geralmente é realizado por meio de modelos autorregressivos. Estudos com curvas de crescimento de bovinos têm mostrado que os modelos com estrutura de erros autorregressivos, apresentaram estimativas mais próximas da realidade e melhor qualidade de ajuste (Mazzini et al., 2005; Mendes et al., 2008).

Apesar de haver um número significativo de estudos sobre o ajuste de modelos de curvas de lactação, principalmente em bovinos, não há referências sobre a utilização de estruturas de erros autorregressivos em tais modelos. O 
emprego dessa técnica estatística pode alterar as estimativas dos coeficientes, e uma vez que estes podem ser usados como características de interesse no processo de seleção, torna-se necessário verificar a influência de tal técnica sobre as estimativas dos parâmetros genéticos destes coeficientes.

Tendo em vista esse aspecto, o presente trabalho teve o objetivo de verificar a influência da autocorrelação residual sobre as estimativas de herdabilidade $\left(\mathrm{h}^{2}\right)$ e repetibilidade $(\mathrm{r})$ para produção total de leite (PL) e para os coeficientes a, b e c do modelo de Wood, consequentemente, também estudar tal influência sobre a classificação dos animais, de acordo com os valores genéticos preditos para as características citadas.

\section{MATERIAL E MÉTODOS}

O controle de leite, registrado semanalmente, foi proveniente de cabras sob ordenha mecânica, realizada duas vezes ao dia, e mantidas em baias coletivas sob sistema de estabulação livre.

Os dados foram coletados de 1997 a 2008. O banco de dados inicial foi composto por 84.377 registros de produção de leite no dia do controle (PLDC) provenientes de 2.706 lactações, porém foram consideradas somente as lactações até a sexta ordem, com idades das cabras ao parto entre 11 e 91 meses, número de controles na lactação entre 19 e 51, e duração da lactação de 199 a 331 dias de cabras com uma ou duas crias. Após estas restrições no arquivo de dados, o modelo de Wood foi ajustado individualmente a 1.069 lactações de cabras das raças Alpina e Saanen e sem raça definida, de três formas diferentes, tendo em vista a presença de resíduos autocorrelacionados e sua posterior modelagem.

$\mathrm{Na}$ primeira, desconsideraram-se as possíveis autocorrelações, ou seja, foram assumidos erros independentes (EI) nos ajustes do modelo de Wood a todas as lactações. Na segunda, o modelo Wood com estrutura de erros autorregressivos de primeira ordem (AR1) foi ajustado a todas as lactações. E na terceira, nomeada de EI-AR1, foi ajustado o modelo com estrutura de erros AR1 somente para as lactações que apresentaram autocorrelação de primeira ordem significativa por meio do teste de Durbin-Watson, sendo que, para as demais, foi adotado o modelo com EI.

A estimação dos coeficientes do modelo de Wood foi feita pelo procedimento PROC MODEL do software SAS (Statistical Analysis System, 2003), que utiliza o método dos quadrados mínimos ordinários via processo iterativo de Gauss-Newton. Os modelos de Wood, assumindo EI e erros AR1, são apresentados, respectivamente, pelas expressões:

$\mathrm{y}_{\mathrm{t}}=a \mathrm{t}^{\mathrm{b}} \exp ^{-\mathrm{ct}}+\mathrm{e}_{\mathrm{t}}(1)$ e $\mathrm{y}_{\mathrm{t}}=a \mathrm{t}^{\mathrm{b}} \exp ^{-\mathrm{ct}}+\varphi_{1} \mathrm{e}_{\mathrm{t}-1}+\mathrm{e}_{\mathrm{t}} \quad$ (2), em que:

$\mathrm{y}_{\mathrm{t}}$ é a produção de leite no tempo t; a, b e c são, respectivamente, os coeficientes associados com a produção média no início da lactação, a taxa média de ascensão da produção até atingir a produção máxima e a taxa média de declínio da produção após atingir a produção máxima; $\mathrm{e}_{\mathrm{t}-1}$ é o resíduo no tempo t-1; $\varphi_{1}$ é o coeficiente autorregressivo de primeira ordem; e $e_{t}$ é o resíduo no tempo t, $\mathrm{e}_{\mathrm{t}} \sim \operatorname{NID}\left(0, \sigma^{2}\right)$.

O fato de se utilizar ajustes individuais possibilitou a obtenção de arquivos de dados contendo as estimativas dos coeficientes para cada animal. Estes arquivos caracterizam-se pelas três diferentes estruturas residuais consideradas, EI, AR1 e EI-AR1. Em cada um desses arquivos, além das estimativas dos coeficientes, considerou-se também a produção total de leite (PL), a qual foi estimada pela fórmula:

$$
\mathrm{PL}=\left(\operatorname{pldc}_{1} * \mathrm{I}_{1}\right)+\sum_{\mathrm{i}=1}^{\mathrm{n}}\left[\left(\frac{\text { pldc }_{\mathrm{i}}+\mathrm{pldc}_{\mathrm{i}+1}}{2}\right) * \mathrm{I}_{\mathrm{i}, \mathrm{i}+1}\right] \text {, }
$$

em que: PL é a produção de leite total da lactação (kg); pldc $_{1}$ é a produção de leite no primeiro controle leiteiro (kg); $\mathrm{I}_{1}$ é o intervalo, em dias, do início da lactação ao primeiro controle; pldc $_{\mathrm{i}}$ é a produção de leite no dia do controle leiteiro i (kg); pldc $_{\mathrm{i}+1}$ é a produção de leite no controle seguinte (kg); $\mathrm{I}_{\mathrm{i}, \mathrm{i}+1}$ é o intervalo, em dias, entre dois controles consecutivos.

Uma informação importante a respeito desses conjuntos de dados é a de que, para a realização das avaliações genéticas, foram utilizadas apenas as lactações em comum às três situações de estrutura residual, totalizando 675 lactações. Dessa forma, espera-se que as diferenças nas estimativas dos parâmetros genéticos sejam 
atribuídas apenas às diferentes estruturas residuais adotadas. $\mathrm{O}$ arquivo de pedigree referente a esses conjuntos de dados foi composto por 819 animais, dos quais 439 apresentaram registros de produção.

Para as avaliações genéticas, foi utilizado o modelo animal multicaracterístico, considerandose simultaneamente a PL e as estimativas dos três coeficientes do modelo de Wood. No modelo em questão, foram considerados os efeitos aleatórios de animal e de ambiente permanente e os efeitos fixos de número de crias por parto $-1,2-$, grupos genéticos -1 a $14-$, ordem de parto -1 a 6 - e grupo contemporâneo -1 a 15 -, sendo este último definido por ano e estação de parto. Além desses efeitos, foram consideradas como covariáveis a duração da lactação - linear - e a idade da cabra ao parto - linear e quadrática.
O modelo descrito anteriormente é representado matricialmente por:

$\mathrm{y}=\mathrm{Xb}+\mathrm{Zg}+\mathrm{Wp}+\varepsilon$, em que:

y é o vetor de observações (PL, a, b, c); X, Z e W são, respectivamente, as matrizes de incidência dos efeitos fixos, aleatórios genéticos aditivos e aleatórios de ambiente permanente; b, g e p são, respectivamente, os vetores de parâmetros referentes aos efeitos fixos, aos valores genéticos aditivos e ao ambiente permanente; e $\varepsilon$ é o vetor de erros aleatórios. Para os efeitos aleatórios, foram assumidas as seguintes distribuições: $\varepsilon \sim$ $\mathrm{N}(0, \mathrm{R}), \mathrm{g} \sim \mathrm{N}(0, \mathrm{G})$ e $\mathrm{p} \sim \mathrm{N}(0, \mathrm{P})$.

As equações de modelos mistos (MME) para o modelo apresentado são dadas por:

$$
\left[\begin{array}{ccc}
X^{\prime} R^{-1} X & X^{\prime} R^{-1} Z & X^{\prime} R^{-1} W \\
Z^{\prime} R^{-1} X & Z^{\prime} R^{-1} Z+G^{-1} & Z^{\prime} R^{-1} W \\
W^{\prime} R^{-1} X & W^{\prime} R^{-1} Z & W^{\prime} R^{-1} W+P^{-1}
\end{array}\right]\left[\begin{array}{c}
b^{0} \\
\hat{g} \\
\hat{p}
\end{array}\right]=\left[\begin{array}{c}
X^{\prime} R^{-1} y \\
Z^{\prime} R^{-1} y \\
W^{\prime} R^{-1} y
\end{array}\right] \text {, em que: }
$$

$\mathrm{G}=\mathrm{A} \otimes \mathrm{G}_{0}$, em que A é a matriz do numerador dos coeficientes de parentesco de Wright entre os animais, $\mathrm{G}_{0}$ é a matriz de variância e covariância genética aditiva entre as características;

$\mathrm{P}=\mathrm{I} \otimes \mathrm{P}_{0}$, em que I é a matriz identidade, $\mathrm{P}_{0}$ é matriz de variâncias e covariâncias de efeito de ambiente permanente;

$\mathrm{R}=\mathrm{I} \otimes \mathrm{R}_{0}$, em que $\mathrm{R}_{0}$ é a matriz de variâncias e covariâncias residuais entre as características e $\otimes$ é o operador de produto direto.

O modelo apresentado foi ajustado separadamente a cada um dos três conjuntos de dados considerados, e tal ajuste foi efetuado por meio do software REMLF90, descrito por Misztal (2002), utilizando-se o método da máxima verossimilhança restrita via algoritmo de maximização da esperança.

\section{RESULTADOS E DISCUSSÃO}

Na Tab. 1 apresentam-se as estimativas da herdabilidade $\left(\mathrm{h}^{2}\right)$ e repetibilidade (r) para PL e para as estimativas dos coeficientes do modelo de Wood - a, b e c -, nas três situações de estrutura residual. Os valores de $h^{2}$ e $r$ para PL foram consistentes com os relatados na literatura (Guimarães et al., 2006), e para as estimativas de a, b e c, a magnitude destes valores $-h^{2}$ e $r-$ informam que a seleção baseada apenas na forma da curva de lactação seria de baixa eficiência.

Com relação a esses parâmetros genéticos, Guimarães et al. (2006), em um estudo com curvas de lactação de cabras predominantemente das raças Alpina e Saanen, estimaram $\mathrm{h}^{2}$ e r para produção de leite em 0,47 e 0,61, respectivamente. Nesse mesmo estudo, também foi constatado que os valores de $h^{2}$ e $r$ para os coeficientes da curva de lactação, obtida segundo o modelo de Nelder (1966), foram muito baixos - 0,01 e 0,01 para a; 0,04 e 0,12 para b e 0,23 e 0,23 para c - , sendo que apenas o coeficiente c, que se refere à persistência da lactação, apresentou valor de média magnitude. Segundo os autores, a seleção de fêmeas para lactação mais persistente poderia ser efetiva. Observa-se que a estratégia em que houve modelagem dos resíduos por meio da estrutura EI - AR1 levou às menores estimativas de $\mathrm{h}^{2}$ e $\mathrm{r}$ para as estimativas dos coeficientes do modelo de Wood (Tab. 1). 
Efeito da autocorrelação residual...

Tabela 1. Estimativas da herdabilidade $\left(\mathrm{h}^{2}\right)$ e repetibilidade (r) para produção total de leite (PL) e para os coeficientes do modelo de Wood (a, b e c), nas três situações de estrutura residual

\begin{tabular}{ccccccc}
\hline \multirow{2}{*}{ Característica } & \multicolumn{7}{c}{ Estrutura residual } \\
\cline { 2 - 7 } & \multicolumn{2}{c}{ EI } & \multicolumn{2}{c}{ AR1 } & \multicolumn{2}{c}{ EI - AR1 } \\
\cline { 2 - 7 } & $\mathrm{h}^{2}$ & $\mathrm{r}$ & $\mathrm{h}^{2}$ & $\mathrm{r}$ & $\mathrm{h}^{2}$ & $\mathrm{r}$ \\
$\mathrm{n}$ PL & 0,3653 & 0,4678 & 0,3556 & 0,4648 & 0,3321 & 0,4838 \\
$\mathrm{~b}$ & 0,0654 & 0,1373 & 0,0619 & 0,1333 & 0,0351 & 0,1277 \\
$\mathrm{c}$ & 0,1251 & 0,1771 & 0,1119 & 0,1582 & 0,0501 & 0,1453 \\
\hline & 0,0769 & 0,1666 & 0,0629 & 0,1574 & 0,0279 & 0,0925 \\
\hline
\end{tabular}

EI, AR1 e EI-AR1 correspondem, respectivamente, às estruturas de erros independentes, autorregressivos de primeira ordem e autorregressivos de primeira ordem somente para as lactações nas quais se constatou autocorrelação significativa.

Na Tab. 2, apresentam-se as estimativas das correlações genéticas e de ambiente permanente entre as características avaliadas - PL, a, b e c -, de acordo com as diferentes estruturas residuais utilizadas - EI, AR1 e EI-AR1 - no ajuste do modelo de Wood. As estruturas residuais afetaram a magnitude de algumas correlações de ambiente permanente e genéticas entre as características avaliadas, sendo observada também alteração no sinal da correlação de ambiente permanente entre os coeficientes b e c, quando utilizada a estrutura residual EI-AR1. As correlações residuais foram semelhantes, no entanto todas elas apresentaram menor magnitude quando foi utilizada a estrutura residual EI - AR1.

Tabela 2. Estimativas das correlações genéticas (acima da diagonal) e de ambiente permanente (abaixo da diagonal) entre produção de leite total (PL) e os parâmetros do modelo de Wood (a, b e c) nas situações de estrutura residual EI, AR1 e EI-AR1

\begin{tabular}{|c|c|c|c|c|}
\hline \multicolumn{5}{|c|}{ EI } \\
\hline & $\mathrm{a}$ & $\mathrm{b}$ & C & PL \\
\hline $\mathrm{a}$ & & $-0,54$ & $-0,50$ & 0,33 \\
\hline $\mathrm{b}$ & $-0,94$ & & 0,94 & 0,60 \\
\hline $\mathrm{C}$ & 0,49 & $-0,41$ & & 0,49 \\
\hline PL & $-0,35$ & 0,50 & $-0,74$ & \\
\hline \multicolumn{5}{|c|}{ AR1 } \\
\hline & $\mathrm{a}$ & b & C & PL \\
\hline $\mathrm{a}$ & & $-0,55$ & $-0,39$ & 0,25 \\
\hline b & $-0,90$ & & 0,87 & 0,63 \\
\hline c & 0,43 & $-0,32$ & & 0,53 \\
\hline PL & $-0,20$ & 0,41 & $-0,71$ & \\
\hline \multicolumn{5}{|c|}{ EI-AR1 } \\
\hline & $\mathrm{a}$ & $\mathrm{b}$ & C & PL \\
\hline $\mathrm{a}$ & & $-0,63$ & $-0,37$ & 0,50 \\
\hline b & $-0,81$ & & 0,81 & 0,12 \\
\hline c & $-0,22$ & 0,58 & & 0,05 \\
\hline PL & 0,09 & 0,22 & $-0,21$ & \\
\hline
\end{tabular}

EI, AR1 e EI-AR1 correspondem, respectivamente, às estruturas de erros independentes, autorregressivos de primeira ordem e autorregressivos de primeira ordem somente para as lactações nas quais se constatou autocorrelação significativa.

As diferentes estruturas residuais alteraram a classificação dos animais para todas as características estudadas, sendo que a classificação na situação de erros EI - AR1 foi a que mais divergiu da classificação nas demais situações. No entanto, como as estimativas de $h^{2}$ e r para os coeficientes de Wood foram muito baixas (Tab. 1), é apresentada apenas a comparação da classificação dos animais, nas três situações de estrutura residual, de acordo com os valores genéticos estimados para PL.

Apesar de a PL, conforme a fórmula em que foi estimada, depender apenas da produção no dia de 
controle leiteiro e do intervalo entre os controles, houve alteração na classificação dos animais de acordo com seus valores genéticos para PL, possivelmente porque, na análise multicaracterística, estes foram influenciados pelas correlações de PL com a, b e c.

As porcentagens de cabras e de reprodutores selecionados em comum para PL nas situações de erros AR1 e EI - AR1, em relação à situação de erros EI, de acordo com a análise multicaracterística de PL com a, b e c, são apresentadas nas Tab. 3 e 4 . Ao se selecionar um menor número de cabras, ou seja, adotando-se maior intensidade de seleção, ocorreu menor coincidência de animais selecionados nas situações de erros AR1 e EI - AR1 com aqueles selecionados na situação de erros EI. Logo, quanto maior a intensidade de seleção, maior é o erro de classificação ao não considerar a autocorrelação residual na avaliação genética, quando esta estiver presente.

Na classificação em comum dos reprodutores, não foi observada relação da intensidade de seleção e erro de classificação ao não se considerar a autocorrelação residual, como na classificação das cabras, principalmente na porcentagem de seleção em comum nas situações de EI e AR1. Isso pode ter ocorrido em razão de o número de reprodutores avaliados ter sido muito reduzido em comparação ao número de cabras, já que foram estimados valores genéticos para 58 machos e 761 fêmeas. Portanto, a seleção dos animais pode ser modificada pela presença da autocorrelação residual, se esta estiver presente e não for considerada nas avaliações genéticas.

Tabela 3. Cabras selecionadas em comum em diferentes situações de seleção para produção de leite nas situações de estrutura residual AR1 e EI - AR1, em relação à EI

\begin{tabular}{ccc} 
Número de cabras & \multicolumn{2}{c}{ Porcentagens de seleção em comum } \\
\cline { 2 - 3 } selecionadas & EI e AR1 & EI e EI - AR1 \\
\cline { 2 - 3 } 38 & 87,5 & 75,0 \\
76 & 92,1 & 73,7 \\
152 & 96,0 & 76,3 \\
304 & 95,4 & 73,7 \\
457 & 96,7 & 78,6 \\
609 & 98,0 & 86,0 \\
\hline
\end{tabular}

EI, AR1 e EI-AR1 correspondem, respectivamente, às estruturas de erros independentes, autorregressivos de primeira ordem e autorregressivos de primeira ordem somente para as lactações nas quais se constatou autocorrelação significativa.

Tabela 4. Reprodutores selecionados em comum em diferentes situações de seleção para produção de leite nas situações de estrutura residual AR1 e EI - AR1, em relação à EI

\begin{tabular}{ccc}
\hline \multirow{2}{*}{$\begin{array}{c}\text { Número de reprodutores } \\
\text { selecionados }\end{array}$} & \multicolumn{2}{c}{ Porcentagens de seleção em comum } \\
\cline { 2 - 3 } & EI e AR1 & EI e EI - AR1 \\
\hline 3 & 100 & 66,7 \\
12 & 100 & 83,3 \\
23 & 91,7 & 75,0 \\
35 & 100 & 82,6 \\
46 & 97,1 & 80,0 \\
\hline
\end{tabular}

EI, AR1 e EI-AR1 correspondem, respectivamente, às estruturas de erros independentes, autorregressivos de primeira ordem e autorregressivos de primeira ordem somente para as lactações nas quais se constatou autocorrelação significativa.

No presente estudo, a situação de erros EI - AR1 proporcionou as menores estimativas para $\mathrm{h}^{2}, \mathrm{r}$ e valores genéticos. No entanto, as estimativas de maior valor obtidas na situação de erros independentes não são necessariamente melhores, pois foram estimadas sem considerar a autocorrelação residual presente em algumas lactações. 


\section{CONCLUSÕES}

A seleção dos animais pode ser prejudicada pela presença da autocorrelação residual, se esta estiver presente e não for considerada nas avaliações genéticas. O uso de resíduos autorregressivos nas lactações com autocorrelação residual significativa apresentase como uma alternativa para contornar este problema. As respostas à seleção para o formato da curva de lactação, mediada pelos coeficientes do modelo de Wood, seriam pequenas, devido às baixas estimativas dos parâmetros genéticos para estas características.

\section{REFERÊNCIAS BIBLIOGRÁFICAS}

FARO, L.E.; ALBUQUERQUE, L.G. Comparação de alguns modelos matemáticos para o ajuste às curvas de lactação individuais de vacas da raça Caracu. Arq. Bras. Med. Vet. Zootec., v.54, p.295-302, 2002. Disponível em $<$ http://www.scielo.br/scielo.php?script=sci_arttext\&p id=S0102-09352002000300013\&lng=en\&nrm=iso>. Acessado em: 01 Mai. 2010.

GROSSMAN, M.; HARTZ, S.M.; KOOPS, W.P. Persistency of lactation yield: a novel approach. $J$. Dairy Sci., v.82, p.2192-2197, 1999.

GUIMARÃES, V.P.; RODRIGUES, M.T.; SARMENTO, J.L.R. et al. Utilização de funções matemáticas no estudo da curva de lactação em caprinos. Rev. Bras. Zootec., v.35, p.535-543, 2006.

JOHANSSON, I.; RENDEL, J. Genética y mejora animal. Zaragoza: Acribia, 1972. 567p.
MAZZINI, A.R.A.; MUNIZ, J.A.; SILVA, F.F. et al. Curva de crescimento de novilhos Hereford: heterocedasticidade e resíduos autorregressivos. Cienc. Rural, v.35, p.422-427, 2005.

MENDES, P.N.; MUNIZ, J.A.; SILVA, F.F. et al. Modelo logístico difásico no estudo do crescimento de fêmeas da raça Hereford. Cienc. Rural, v.38, p.1984-1990, 2008.

MISZTAL, I. REMLF90 manual. 2002. Disponível em: <http://nce.ads.uga.edu/ ignacy/numpub/blupf90/docs/remlf90.pdf $>$. Acessado em: 15 nov. 2008.

NELDER, J.A. Inverse polynomials a useful group of multi-factor response functions. Biometrics, v.22, p.128-141, 1966.

OLIVEIRA, H.T.V.; REIS, R.B.; GLÓRIA, J.R. et al. Curvas de lactação de vacas F1 Holandês-Gir ajustadas pela função gama incompleta. Arq. Bras. Med. Vet. Zootec., v.59, p.233-238, 2007.

SÖLKNER, J.; FUCHS, W. A comparison of different measures of persistency with special respect to variation of test-day milk yields. Liv. Prod. Sci., v.16, p.305-319, 1987.

SOUZA, G.S. Introdução aos modelos de regressão linear e não linear. Brasília: EmbrapaSPI/Embrapa - SEA, 1998. 489p.

STATISTICAL analysis system user's guide. Version 9.1 Cary: SAS Institute, 2003.

WOOD, P.D.P. Algebraic model of the lactation curve in cattle. Nature, v.216, p.164-165, 1967. 